\title{
Oral mucositis in cancer treatment: Natural history, prevention and treatment (Review)
}

\author{
MARIA INES DA CRUZ CAMPOS ${ }^{1}$, CELSO NEIVA CAMPOS ${ }^{2}$, \\ FERNANDO MONTEIRO AARESTRUP ${ }^{3}$ and BEATRIZ JULIÃO VIEIRA AARESTRUP ${ }^{4}$ \\ ${ }^{1}$ Postgraduate Programme in Brazilian Health; ${ }^{2}$ Department of Dental Clinic, Dental School; \\ ${ }^{3}$ Laboratory of Immunopathology and Clinical Immunology; ${ }^{4}$ Department of Morphology (Histology and Embryology), \\ Institute of Biological Sciences Research, Federal University of Juiz de Fora, Minas Gerais, Brazil
}

Received October 3, 2013; Accepted January 20, 2014

DOI: $10.3892 / \mathrm{mco} .2014 .253$

\begin{abstract}
Oral mucositis is a condition that is characterized by ulcerative lesions in the mucosa of patients undergoing radiotherapy or chemotherapy. Oral mucositis is currently considered to be the most severe complication of anticancer therapy, affecting $40-80 \%$ of patients undergoing chemotherapy and almost all those undergoing radiotherapy of the head and neck. Although they do not prevent lesions from appearing, drugs for the treatment of oral mucositis are required to minimize its clinical aggressiveness and improve the nutritional status, hydration and quality of life of the affected patients. Furthermore, the prevention and control of oral ulcers is crucial for cancer prognosis, since the establishment of severe lesions may lead to temporary or permanent treatment discontinuation and compromise cancer control. The objective of this study was to present a review on this condition, its causes and its treatment to professional clinical dentists, in order to help minimize patient suffering. A search was conducted through PubMed, Lilacs and MedLine, to retrieve related articles published between 1994 and 2013.
\end{abstract}

\section{Contents}

1. Background/literature review

2. Oral care prior to chemotherapy or radiotherapy

3. Treatments for oral mucositis

4. Conclusions

Correspondence to: $\mathrm{Dr}$ Beatriz Julião Vieira Aarestrup, Department of Morphology (Histology and Embryology), Institute of Biological Sciences, Federal University of Juiz de Fora, s/n Jose Lourenco Kelmer Street, University Campus, Sao Pedro District, Juiz de Fora, Minas Gerais 36036-900, Brazil

E-mail: beatrizaarestrup@gmail.com

Key words: oral mucositis, prevention and control, therapy, pathology

\section{Background/literature review}

This study aimed to present a literature review of oral mucositis, its causes and its treatment to clinical dentists, in order to help minimize patient suffering. A search was conducted through PubMed, Lilacs and MedLine, to retrieve related articles published between 1994 and 2013. The search terms used were oral mucositis, prevention and control, pharmacological effects and immunosuppressive agents. Of the retrieved articles, a total of 29 were finally selected for inclusion in this literature review.

The term 'mucositis' emerged in 1980 to describe ulcerative lesions of the oral mucosa in patients undergoing radiotherapy or chemotherapy. Oral mucositis is currently considered to be the most severe non-hematological complication of anticancer therapy, affecting 40-80\% of patients undergoing chemotherapy and almost all those undergoing radiotherapy of the head and neck (1-4).

In patients treated with radiotherapy, mucositis is considered to be the most important acute side effect in the oral cavity, whereas in patients undergoing chemotherapy, oral mucositis is the most common cause of morbidity, with a higher incidence among patients with solid tumors receiving myelosuppressive chemotherapy. The oral lesions lead to a considerably decreased quality of life in these patients, due to dysphagia (difficulty in feeding) with solid and liquid food, dysarthria (poor coordination of the speech muscles) and odynophagia (pain or burning sensation on swallowing); in addition, the lesions may represent a gateway for opportunistic infections (5-10).

The cytotoxic effect of chemotherapeutic agents on the oral mucosa is discreet during the first days of treatment, gradually becoming more severe from the end of the first week onwards (usually 7-10 days). Two weeks after the end of treatment, the oral lesions resolve $(1,11,12)$.

Macroscopically, oral mucositis initially presents as an erythematous plaque that progresses to ulcerations, with a natural history that is affected by oral cavity-specific factors, such as xerostomia and colonization by opportunistic microbiota. Extrinsic factors, such as smoking and alcohol abuse during radiotherapy and/or chemotherapy, may lead to the development of exuberant lesions and, when associated with fungal infections and poor oral hygiene, mucositis is considered as severe and may require partial or complete 
discontinuation of radiotherapy prior to the completion of the planned protocol, which negatively affects the patient prognosis $(3,4,13)$. Of note, $\sim 11 \%$ of cases of discontinuation and modification of the radiotherapy regimen occur due to the development of severe oral mucositis, with the need of treatment redesign and, in some cases, patient hospitalization (8). In addition to the association of oral mucositis with a worse patient prognosis due to the potential discontinuation of chemotherapy and/or radiotherapy, infection of the lesions by opportunistic microorganisms may lead to bacteremia (4).

According to the currently available literature, the symptoms for oral mucositis vary from oral burning to severe pain, spontaneous bleeding that disrupts routine feeding, which may eventually lead to cachexia and parenteral nutrition via a nasogastric tube $(5,14)$. Azevedo et al $(15)$ emphasized that pain is a significant component of oral mucositis, contributing to decreased quality of life for cancer patients.

From a histopathological point of view, the erythematous lesions of the oral mucosa are characterized by atrophy, which is associated with an inflammatory reaction in the lamina propria. With the persistence of the triggering agent -in this case, the secondary action of the chemotherapeutic drug and/or radiotherapy- ulceration occurs in isolated points that eventually converge to form deeper and larger lesions (16). Of note, after exacerbation of the inflammatory reaction, the lesions undergo spontaneous resolution. Several authors attributed the evolution of the local inflammatory reaction at the mucositis site to a systemic condition, particularly the patient's white blood cell count during cancer treatment $(7,14)$.

Sonis (7), through several clinical and experimental observations, proposed that the stomatotoxicity of the drugs used leads to a complex pathophysiological mechanism underlying the development of oral mucositis, with a natural history including 4 biological stages: initial, vascular or inflammatory stage (acute, as triggered by the initial stimulus of the chemotherapeutic agent); epithelial stage (action of the drug on the epithelial cell cycle, resulting in a reduced rate of cell division); ulceration stage (exposure of the lamina propria by epithelial loss); and healing stage (resolution due to cell proliferation and differentiation). Lima et al (17) reported that the healing stage may be associated with the normalization in the number of circulating leukocytes and subsequent control of the oral microbiota.

Subsequently, the natural history of oral mucositis was described in 5 stages, as outlined by the World Health Organization $(9,14)$. Each sequential stage represents the actions of cytokines and growth factors associated with the effect of chemotherapeutic drugs on the epithelium, the oral bacterial flora and the general condition of the patient $(4,12)$ : i) Inflammatory stage, also referred to as initial, vascular or epithelial stage, it occurs due to a direct effect of the chemotherapeutic drug, which stimulates the early release of chemical mediators by neutrophils and interleukin-1 by macrophages and lymphocytes. These pro-inflammatory cytokines trigger a prompt inflammatory response resulting in increased vascular permeability in the subepithelial connective tissue $(12,18)$. The action of the drug is associated with the release of free radicals and inhibits the mitosis of the basal cell layer in the mucosal epithelium by directly damaging cellular DNA. An acute inflammatory reaction occurs and promotes predominant diapedesis of neutrophils that release vasoactive and chemotactic mediators. This epithelial stage is optimal for documentation, specifically for those agents known to affect epithelial cell division in the oral mucosa (drugs promoting DNA synthesis) and starts to develop 4-5 days after the initiation of chemotherapy. This stage may be longer in terms of the development of ulcerative lesions (12). ii) The maximization stage is associated with release and activation of growth factors that control cellular DNA transcription. Cytokines released from keratinocytes, endothelial cells and the cells of the lamina propria enhance cell damage; concomitantly, chemotherapeutic drugs and radiation activate enzymes that increase apoptosis (programmed cell death). iii) The amplification and signaling stage is characterized by a predominance of pro-inflammatory cytokines that have the ability to regulate transcription factors. The levels of certain substances produced by the cells of the lamina propria are increased and enhance cell damage, in addition to amplifying the action of the transcription factors. iv) During the ulceration stage, mucosal integrity is broken and nerve endings are exposed, leading to severe pain. The colonization of the ulcerated surface by oral microflora results in the release of toxins into the tissues, with subsequent release of additional cytokines that cause more tissue damage and lead to a reduced number of neutrophils. Bacteria may also invade the lamina propria and penetrate vascular walls, leading to bacteremia or sepsis. This stage is associated with a deterioration of the patient's health, in addition to an increase in the healthcare cost. The ulcerative stage is considered to be the most significantly symptomatic period. At this stage, superficial destruction of the epithelium with exposure of the lamina propria leads to the dissolution of the protective barrier between the internal and external medium. v) The cure stage is characterized by lesion resolution, with control of the microflora, gradually decreasing inflammatory reaction and predominance of repair stimuli, with the development of granulation tissue and remission of lesions and it is histopathologically associated with local cell proliferation and differentiation. The final events culminate with hemodynamic peripheral restoration and control of the oral bacterial flora. The duration of this stage depends on the patient's systemic condition (12).

The control of oral mucositis has been the focus of several studies, which highlighted the fact that its effective management may allow for more aggressive therapeutic doses and a more effective cancer treatment and improved patient survival $(3,11,12,14)$.

Due to its high incidence and clinical significance in patient prognosis, several studies were conducted to investigate the treatment and prevention of oral mucositis $(11,18-20)$.

Considering the pain associated with mucositis and its effect on the quality of life of the patients, the use of local anesthetics and analgesics was recommended by some authors $(8,21)$. Natural agents with antimicrobial action, such as chamomile, glycerin, sage and myrrh, administered as mouthwashes, are also mentioned in the literature $(4,8)$. However, despite the application of these therapies, alone or with the combined use of antimicrobials, to prevent or reduce the severity of oral mucositis, there are currently no conclusive records on their efficiency or established prescription protocols $(10,18)$.

In addition to the local benefits of treating oral mucositis, it is crucial to improve the nutritional status, hydration and quality of life of the affected patients. Furthermore, the prevention and control of oral mucositis are vital for cancer prognosis, since the establishment of severe lesions may lead 
to temporary or permanent treatment discontinuation and compromise cancer control $(1,4,8)$.

\section{Oral care prior to chemotherapy or radiotherapy}

In order to minimize the consequences of mucositis in cancer patients undergoing chemotherapy, several oral hygiene protocols are applied extensively and involve the treatment of carious lesions and hygiene maintenance. The main objective of these programs is to reduce the metabolic activity of the oral microflora and to prevent or reduce discomfort associated with oral mucositis $(1,2,5,6,11,19)$. Reducing the amount of microorganisms decreases the likelihood of secondary infections of the primary oral lesions (17).

Keefe et al (22) reviewed the literature published between January, 2002 and May, 2005 and created a consensus based on the American Society of Clinical Oncology criteria. Based on these data, the authors published an oral care guide in order to reduce the negative consequences in the oral cavity caused by radiotherapy and/or chemotherapy treatments. Subsequently, new protocols have been suggested to minimize the problems caused by chemotherapy or radiotherapy.

It is advisable that a complete oral examination be performed prior to the initiation of cancer therapy. A preventive oral care program should be followed to reduce the incidence of complications arising from chemotherapy. The pre-chemotherapy protocol should include root scaling and crown prophylaxis, treatment of carious lesions and endodontic treatment, if necessary. In cases of severe dental pathologies, dental extraction should be considered. The preliminary periodontal treatment is also indicated to eliminate potential sites of infection, since chronic periodontal disease is associated with an increased number of microorganisms in the periodontal pockets. Of note, invasive manipulation of the soft tissues during chemotherapy is contraindicated, due to the subsequent development of bacteremia leading to granulocytopenia (22).

A number of oral conditions have been presented and should be taken into consideration as predisposing factors for patient deterioration: poor oral hygiene, caries associated with periapical pathologies and periodontal disease. Local irradiation of the structures around the head and neck may exacerbate the inflammation and affect the integrity of the oral mucosa by altering the supporting structures, the glands and bone (3).

Should there arise a need for dental extraction during cancer treatment, neutropenia (reduced neutrophil count, which may reach 500-1,000/ $\mu$ l) should be controlled and antibiotic prophylaxis is warranted, including Gram-positive microorganisms and anaerobes. The majority of secondary infections in oral mucositis are caused by Candida albicans or type 1 herpes simplex virus and are reported in $\sim 70 \%$ of the patients (3).

\section{Treatments for oral mucositis}

Roopashri and Jayanthi (21) listed various treatment options for oral mucositis, including cryotherapy, the use of helium-neon laser and the construction of radiation fields to protect the oral tissues during irradiation. In addition, a range of mouthwash options are used that possess anti-inflammatory, anesthetic, analgesic, antipyretic and antimicrobial properties. Systemically administered pharmacological agents, such as pentoxifylline, thalidomide and simvastatin, were shown to correlate with the development and severity of all the complications reported in transplants. These drugs were shown to reduce the frequency and severity of major complications, including oral mucositis, in clinical trials $(15,17)$.

Jain et al (23) conducted a study using a hydrogel containing L-glutamine, which was applied to the intraoral lesions with a cotton swab and was shown to be effective in decreasing inflammatory lesion sensitivity.

Cryotherapy is a practice which is commonly indicated for the relief of lesions caused by mucositis. Sucking on small ice cubes and allowing them to circulate around the oral cavity for $\sim 30 \mathrm{~min}$, provides pain relief and may prevent the development of new lesions $(11,24)$.

A new drug, palifermin, appears to stimulate epithelial cell proliferation and differentiation in the mouth and the gastrointestinal tract. However, the benefits provided by the prophylactic use of this drug require verification, since the currently available data in the literature are insufficient for large-scale use. Another reason limiting the applicability of palifermin for the prevention of oral mucositis is its high cost (25-27).

Helium-neon laser therapy, which uses low-intensity laser, is indicated as a pre-treatment to reduce the severity of mucositis in patients undergoing chemotherapy. However, since this type of treatment requires costly equipment and specialized operators, its use is often restricted to a limited number of patients $(19,20,28)$.

Supplemental parenteral glutamine has been investigated for patients who receive chemotherapy. However, there is currently no scientific evidence confirming its benefit in preventing or decreasing the severity of oral mucositis $(1,11)$.

The use of chlorhexidine, hydrogen peroxide, calcium phosphate, or allopurinol in the form of mouthwash are additional options reported in the literature. However, there is no scientific proof of their effectiveness in the management of oral mucositis $(1,29)$.

A diet restricted in acidic, salty or spicy foods may help reduce the painful symptoms of the oral lesions caused by mucositis (28).

One of the most popular choices are oral mucosal protectants, including Gelclair and Orabase. Gelclair is a mucosal adherent alcohol- and anesthetic-free ointment containing maltodextrin and sodium hyaluronidase, which forms a physical barrier on the surface of the affected mucosa, protecting against irritation by food, liquids and even saliva (5).

The topical application of vitamin $\mathrm{E}$ was also investigated and complete resolution of the lesions was reported. However, further studies are required, since this medication was shown to be effective for the treatment of already established lesions, but does not prevent the development of new lesions (2).

\section{Conclusions}

Oral mucositis is a painful consequence of anticancer chemotherapy and/or radiotherapy of the head and neck, developing in $\sim 90 \%$ of patients undergoing this type of treatment. Dentists must be familiar with the necessary interventions, in order to help the patient during the course of the treatment and avoid treatment interruption. Certain measures may help minimize the symptoms associated with oral mucositis; 
however, further research is required, focusing on lesion prevention prior to treatment initiation. Clinical dentists may help the patients by instructing them on oral hygiene and performing minor dental treatments prior to the initiation of chemotherapy or radiotherapy.

\section{References}

1. Dodd MJ, Dibble SL, Miaskowski C, et al: Randomized clinical trial of the effectiveness of 3 commonly used mouthwashes to treat chemotherapy-induced mucositis. Oral Surg Oral Med Oral Pathol Oral Radiol Endod 90: 39-47, 2000.

2. El-Housseiny AA, Saleh SM, El-Masry AA and Allam AA: The effectiveness of vitamin ' $E$ ' in the treatment of oral mucositis in children receiving chemotherapy. J Clin Pediatr Dent 31: 167-170, 2007.

3. NegrinRS,BedardJFandToljanicJA:Oraltoxicity associated with chemoterapy. http://www.uptodate.com/contents/oral-toxicityassociated-with-chemotherapy. Accessed September 14, 2012.

4. Sonis ST: Pathobiology of mucositis. Semin Oncol Nurs 20: 11-15, 2004.

5. Barber C, Powell R, Ellis A and Hewett J: Comparing pain control and ability to eat and drink with standard therapy vs. Gelclair: a preliminary, double centre, randomised controlled trial on patients with radiotherapy-induced oral mucositis. Support Care Cancer 15: 427-440, 2007.

6. Dorr W, Hamilton CS, Boyd T, Reed B and Denham JW: Radiation-induced changes in cellularity and proliferation in human oral mucosa. Int J Radiat Oncol Biol Phys 52: 911-917, 2002

7. Sonis ST: Mucositis as a biological process: a new hypothesis for the development of chemotherapy-induced stomatotoxicity. Oral Oncol 34: 39-43, 1998.

8. Sonis ST: Efficacy of palifermin (keratinocyte growth factor-1) in the amelioration of oral mucositis. Core Evid 4: 199-205, 2010

9. Sonis ST: New thoughts on the initiation of mucositis. Oral Dis 16: 597-600, 2010.

10. Steinmann D, Eilers V, Beynenson D, Buhck H and Fink M: Effect of Traumeel $\mathrm{S}$ on pain and discomfort in radiation-induced oral mucositis: a preliminary observational study. Altern Ther Health Med 18: 12-18, 2012.

11. Kowanko I, Long L, Hodgkinson B and Evans D: The effectiveness of strategies for preventing and treating chemotherapy and radiation induced oral mucositis in patients with cancer: a systematic review. The Joanna Briggs Institute for Evidence Based Nursing and Midwifery: 2, 1998.

12. Pico JL, Avila-Garavito A and Naccache P: Mucositis: its occurrence, consequences, and treatment in the oncology setting. Oncologist 3: 446-451, 1998.

13. Yeoh AS, Gibson RJ, Yeoh EE, et al: A novel animal model to investigate fractionated radiotherapy-induced alimentary mucositis: the role of apoptosis, p53, nuclear factor-kappaB, COX-1, and COX-2. Mol Cancer Ther 6: 2319-2327, 2007.
14. Lalla RV, Sonis ST and Peterson DE: Management of oral mucositis in patients who have cancer. Dent Clin North Am 52: 61-77, 2008.

15. Azevedo IM, Kumakura HS, Alloufa SL, et al: Effect of simvastatin in attenuation of mucositis induced by methotrexate in rats. J Surg Clin Res 1: 22-32, 2010.

16. Peterson DE and D'Ambrosio JA: Nonsurgical management of head and neck cancer patients. Dent Clin North Am 38: 425-445, 1994.

17. Lima V, Vidal FD, Rocha FA, Brito GA and Ribeiro RA: Effects of tumor necrosis factor-alpha inhibitors pentoxifylline and thalidomide on alveolar bone loss in short-term experimental periodontal disease in rats. J Periodontol 75: 162-168, 2004.

18. Silverman S Jr: Diagnosis and management of oral mucositis. J Support Oncol 5: 13-21, 2007.

19. Kuhn A, Porto FA, Miraglia P and Brunetto AL: Low-level infrared laser therapy in chemotherapy-induced oral mucositis: a randomized placebo-controlled trial in children. J Pediatr Hematol Oncol 31: 33-37, 2009.

20. Migliorati C, Hewson I, Lalla RV, et al: Systematic review of laser and other light therapy for the management of oral mucositis in cancer patients. Support Care Cancer 21: 333-341, 2013.

21. Roopashri G and Jayanthi K: Radiotherapy and chemotherapy induced oral mucositis - prevention and current therapeutic modalities. IJDA 2: 174-179, 2010.

22. Keefe DM, Schubert MM, Elting LS, et al: Updated clinical practice guidelines for the prevention and treatment of mucositis. Cancer 109: 820-831, 2007.

23. Jain P, Keservani RK and Dahima R: In-vivo characterization of hydrogel for treatment of chemo-radiotherapy induced oral mucositis. Pharmacol Online 1: 1016-1025, 2010.

24. Peterson DE, Ohrn K, Bowen J, et al: Systematic review of oral cryotherapy for management of oral mucositis caused by cancer therapy. Support Care Cancer 21: 327-332, 2013.

25. Li E and Trovato JA: New developments in management of oral mucositis in patients with head and neck cancer or receiving targeted anticancer therapies. Am J Health Syst Pharm 69: 1031-1037, 2012.

26. Raber-Durlacher JE, von Bultzingslowen I, Logan RM, et al: Systematic review of cytokines and growth factors for the management of oral mucositis in cancer patients. Support Care Cancer 21: 343-355, 2013.

27. Siddiqui MA and Wellington K: Palifermin: in myelotoxic therapy-induced oral mucositis. Drugs 65: 2139-2149, 2005.

28. Gautam AP, Fernandes DJ, Vidyasagar MS, Maiya AG and Vadhiraja BM: Low level laser therapy for concurrent chemoradiotherapy induced oral mucositis in head and neck cancer patients - a triple blinded randomized controlled trial. Radiother Oncol 104: 349-354, 2012.

29. Porta C, Moroni M and Nastasi G: Allopurinol mouthwashes in the treatment of 5-fluorouracil-induced stomatitis. Am J Clin Oncol 17: 246-247, 1994. 